\title{
Auch nach sechs Jahren noch keinen Studienplatz?
}

\author{
Bewerber für medizinische Studiengänge müssen wohl doch bis zu sieben \\ Jahre auf einen Studienplatz warten. Das Oberverwaltungsgericht (OVG) \\ Nordrhein-Westfalen in Münster hat mit mehreren Beschlüssen gegenteilige \\ Entscheidungen des Verwaltungsgerichts (VG) Gelsenkirchen aufgehoben.
}

— Etwa $40 \%$ der Studienplätze in medizinischen Fächern werden durch die Dortmunder Stiftung für Hochschulzulassung vergeben, die übrigen Plätze durch die Hochschulen selbst. Für die Zulassung zum Wintersemester 2011/2012 war bei der Stiftung ein Notenschnitt unter 1,2 notwendig, bei nicht so guten Noten spielt auch die Wartezeit eine Rolle.

\section{Das Grundrecht auf freie Berufswahl wird eingeschränkt}

Mit mehreren Eilbeschlüssen hatte das VG Gelsenkirchen eine unzureichende Gewichtung der Wartezeiten gerügt: Wartezeiten über sechs Jahre seien mit dem Recht auf freie Berufswahl nicht mehr vereinbar. Zudem könnten sich sich Bewerber nicht einmal auf einen bestimmten Studienbeginn einstellen.

Nun wird es aber trotzdem nichts mit einem baldigen Studienbeginn: Die Stif- tung hat beim OVG Beschwerde eingelegt und gleichzeitig beantragt, den Vollzug der VG-Entscheidungen auszusetzen. Diesem Antrag gab das OVG nun statt. Die Gelsenkirchener Beschlüsse würden sich „mit überwiegender Wahrscheinlichkeit als fehlerhaft erweisen".

Ein Anspruch, ausgerechnet nach einer Wartezeit von sechs Jahren zugelassen $\mathrm{zu}$ werden, bestehe nicht, so das OVG. Schließlich sei nach einem weiteren Jahr „eine Zulassung zum Wintersemester 2012/2013 hinreichend wahrscheinlich".

\section{Warten oder gute Noten?}

Die verfassungsrechtlich verbürgte Chance auf den gewünschten Studienplatz sei damit, „wenn auch verspätet“, noch gegeben. Zudem würden wegen überlanger Wartezeiten zugelassene Bewerber andere mit besseren Abiturnoten

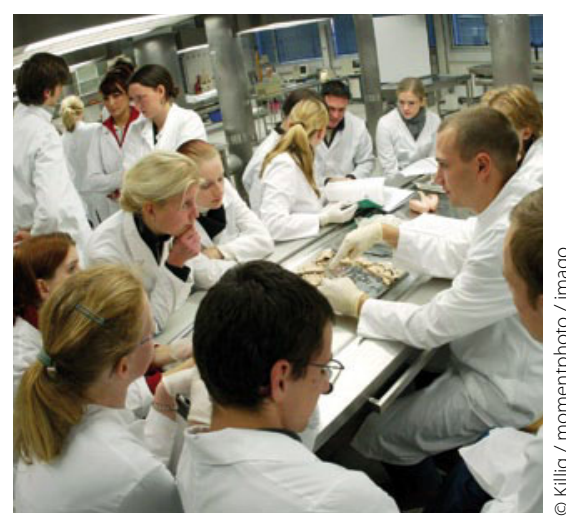

Medizin-Studienplätze sind heiß begehrt.

verdrängen. Die Rechte beider Gruppen auszubalancieren, sei aber letztlich eine Entscheidung der Politik.

Die Beschlüsse sind zunächst unanfechtbar. Erst im anschließenden Hauptverfahren ist dann auch eine Revision zum Bundesverwaltungsgericht möglich. Bis dahin hat aber sicherlich auch das Wintersemester 2012/2013 längst begonnen.

MWO =

Az.: 13 B 1214/11, 13 B 1215/11, 13 B 1216/11, 13 B 1217/11 und 13 B 1218/11

\section{Krebsvorsorge im Büro ist im Kommen}

Betriebs- und Werksärzte können Männerleben retten. Ohne sie wäre es um die Krebsfrüherkennung schlechter bestellt, sagen Experten.

- Arbeitsmedizinern kommt bei der Früherkennung von Krebserkrankungen eine steigende Bedeutung zu, meint Prof. Eckhard Wilhelm Breitbart, Mitglied im Fachausschuss Krebsfrüherkennung der Deutschen Krebshilfe.
„In den Betrieben kommen die Ärzte mit Bevölkerungs- und Altersgruppen in Kontakt, die sie sonst nur schwer erreichen", sagte Breitbart beim Deutschen Betriebsärzte-Kongress in Bonn. „In den Betrieben kommen wir auch an die Männer heran, die bekanntlich Präventionsmuffel sind."

Die Deutsche Krebshilfe arbeitet seit rund sechs Jahren mit dem Verband Deutscher Betriebs- und Werksärzte
(VDBW) zusammen. Wichtig seien etwa gemeinsame Früherkennungsaktionen z. B. für Darmkrebs.

VDBW-Vizepräsidentin Dr. Anette Wahl-Wachendorf betonte die zentrale Rolle der Betriebsärzte beim Entdecken von Erkrankungen: „20-30\% der männlichen Beschäftigten haben keinen Hausarzt", sagte sie.

ISS 